\title{
A Case Series of Unusual or Infrequent Locations of Breast Cancer Metastases
}

\author{
Amanda Ling Fung Liew ${ }^{1} \quad$ Ern Yu Yu $u^{2,3,4}$ Niketa Chotai ${ }^{5,6}$ \\ ${ }^{1}$ Division of Oncologic Imaging, National Cancer Centre Singapore, \\ 11 Hospital Crescent, Singapore \\ 2Department of General Surgery, Tan Tock Seng Hospital, 11 Jln Tan \\ Tock Seng, Singapore \\ ${ }^{3}$ Lee Kong Chian School of Medicine, Nanyang Technological \\ University, 11 Mandalay Rd, Singapore \\ ${ }^{4}$ Institute of Molecular and Cell Biology, Agency for Science, \\ Technology and Research (A*STAR), 61 Biopolis Dr, Singapore \\ ${ }^{5}$ Department of Diagnostic Radiology, Tan Tock Seng Hospital, 11 \\ Jln Tan Tock Seng, Singapore \\ 6RadLink Diagnostic Imaging, Singapore, 08-08, 290 Orchard Road, \\ Singapore \\ Indian J Radiol Imaging 2021;31:366-372.
}

\begin{abstract}
Address for correspondence Niketa Chotai, MBBS, MD, DNB, FRCR, RadLink Diagnostic Imaging, 08-08, 290 Orchard Road, Singapore 238859 (e-mail: niketachotai@gmail.com).
\end{abstract}

\begin{abstract}
Keywords

- adults

- breast

- metastases

Breast cancer metastasis to unusual locations may present as a diagnostic challenge. Recognizing the different manifestations of metastatic breast disease can help to guide patient treatment and assist in disease prognosis. The purpose of this article is to illustrate the imaging findings of some of the unusual or infrequent locations of breast metastases through a series of interesting cases and to revisit this uncommon clinical issue.
\end{abstract}

\section{Introduction}

About $7 \%$ of breast cancers are metastatic at diagnosis. The exact incidence of metastatic recurrence is unknown but is estimated to range between 20 and $30 \% .{ }^{1}$ Although the management of metastatic breast disease has evolved with recent advances in treatment, its impact on the overall survival rate remains inconsistent, with mixed results reported in literature. Some reports showed that between 3 and $30 \%$ of patients with distant metastases can achieve long-term survival with multimodality therapy, while others have not been able to report an overall survival in metastatic breast cancer. ${ }^{1,2}$ At present, the evidence shows that the total burden of systemic disease has a greater influence on the survival rate of patients with metastatic disease., ${ }^{1,2}$ As such, it is important to recognize the different manifestations of metastatic breast disease for the accurate assessment of disease extent.

It has long been established that the common locations of breast metastases are bone, lung, brain, and liver. ${ }^{1,3}$ This article presents a series of interesting cases of breast metastases in unusual or infrequent locations such as the gastrointestinal tract (GIT), pancreas, spleen, ureter, ovaries, and orbits

published online

July 30,2021
DOI https://doi.org/

$10.1055 / \mathrm{s}-0041-1734338$ ISSN 0971-3026 and focuses on their imaging features. All cases included have either histopathological diagnosis or evidence of treatment response to confirm the etiology.

\section{Case Series}

\section{Gastrointestinal Tract-5 Cases \\ Case 1}

A 57-year-old lady presented with a stage- 4 metastatic invasive adenocarcinoma of the breast at diagnosis. She developed abdominal distention while on palliative chemotherapy. Contrast-enhanced computed tomography (CT) scan of the abdomen and pelvis revealed focal enhancement and thickening in the gastric fundus ( - Fig. 1 ). Biopsy of the stomach revealed metastatic invasive adenocarcinoma of the breast to the stomach.

\section{Case 2}

A 50-year-old lady presented with a prior history of stage- 1 invasive lobular carcinoma of the breast. She presented with abdominal pain 9 years after treatment. Contrast-enhanced CT scan of the abdomen and (c) 2021. Indian Radiological Association.

This is an open access article published by Thieme under the terms of the Creative Commons Attribution-NonDerivative-NonCommercial-License, permitting copying and reproduction so long as the original work is given appropriate credit. Contents may not be used for commercial purposes, or adapted, remixed, transformed or built upon. (https://creativecommons.org/licenses/by-nc-nd/4.0/).

Thieme Medical and Scientific Publishers Private Ltd. A-12, Second Floor, Sector -2, NOIDA -201301, India 


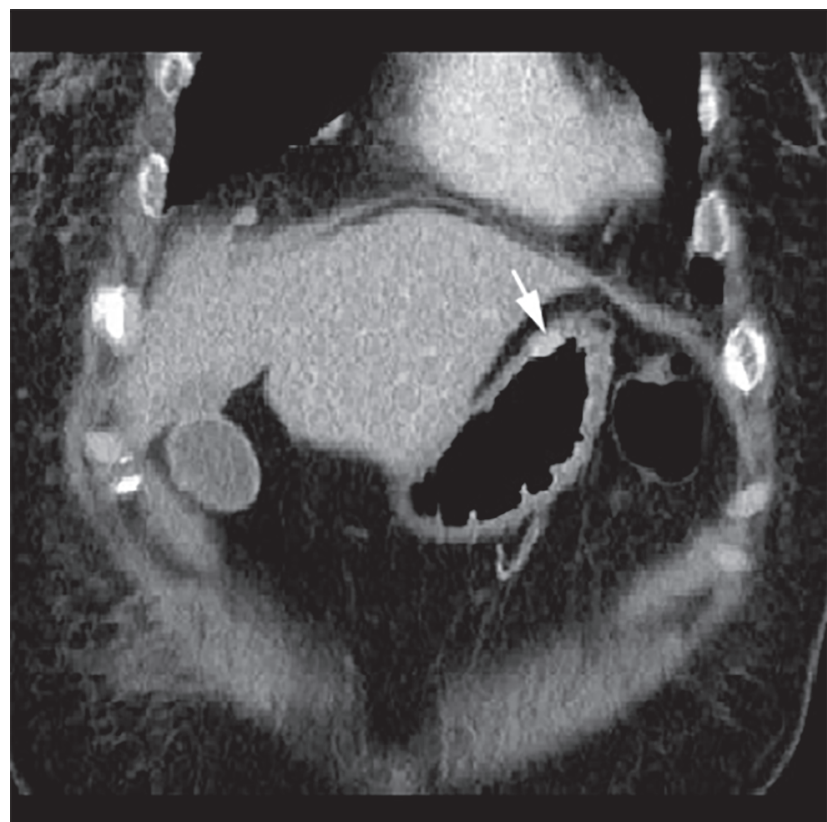

Fig. 1 Histologically proven metastasis of invasive breast adenocarcinoma breast to the stomach. Computed tomography shows focal enhancement and thickening in the gastric fundus (arrow); a rare presentation as compared with the more common linitis plastica for stomach metastases.

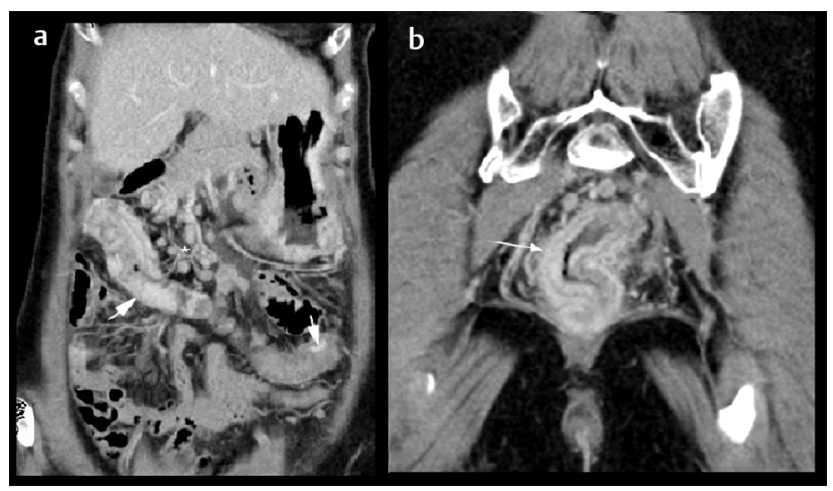

Fig. 2 Histologically proven metastasis of invasive lobular breast carcinoma to the colon. Computed tomography (A) shows discontinuous hyperenhancing irregular thickening of the hepatic flexure and transverse colon (arrows) and enlarged mesenteric lymph nodes (asterisk) and (B) thickening of the rectum (arrow). Multiplicity favors metastases.

pelvis revealed discontinuous irregular wall thickening of the hepatic flexure and transverse colon and rectum (-Fig. 2). Biopsy of the transverse colon and rectum showed metastatic invasive lobular breast carcinoma to the affected sites.

\section{Case 3}

A 63-year-old lady with stage-4 metastatic invasive lobular carcinoma of the breast at diagnosis developed constipation while on palliative chemotherapy. Contrast-enhanced magnetic resonance imaging (MRI) scan of the rectum showed markedly T2-hypointense circumferential thickening of the serosa and muscularis propria of the rectum with sparing of the mucosa ( - Fig. 3). Biopsy of the rectum showed metastatic invasive lobular breast carcinoma to the rectum.

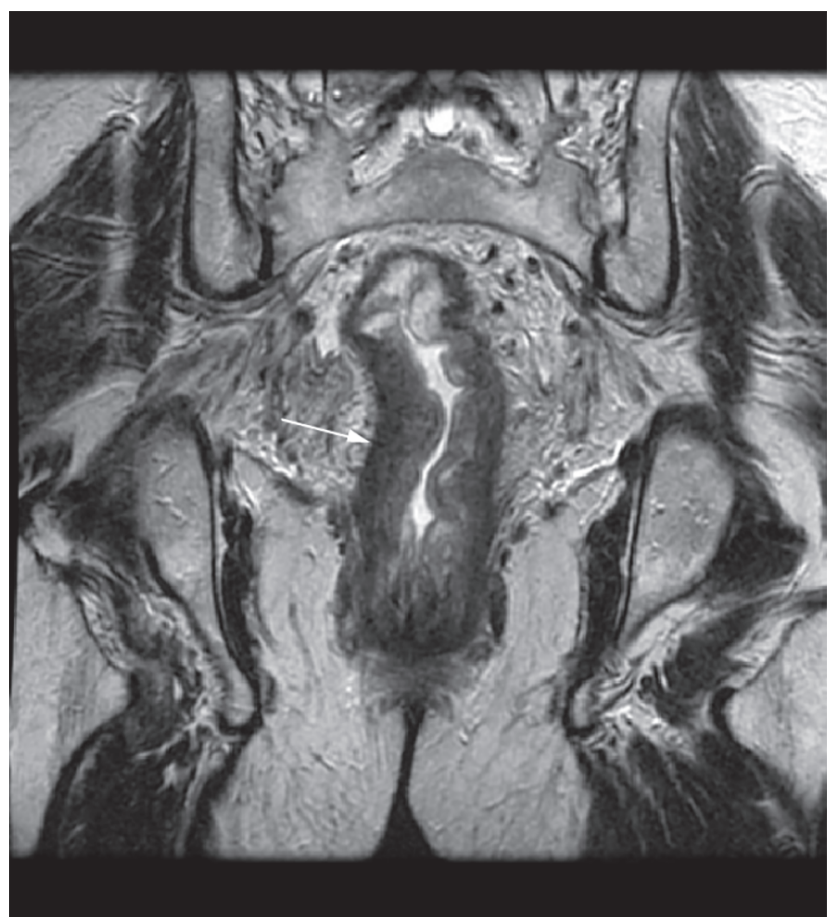

Fig. 3 Histologically proven metastasis of invasive lobular breast carcinoma to the rectum. Coronal T2-weighted magnetic resonance imaging shows markedly T2-hypointense circumferential thickening of the serosa and muscularis propria of the rectum (arrow) with characteristic sparing of the mucosa, differentiating it from primary rectal carcinoma.

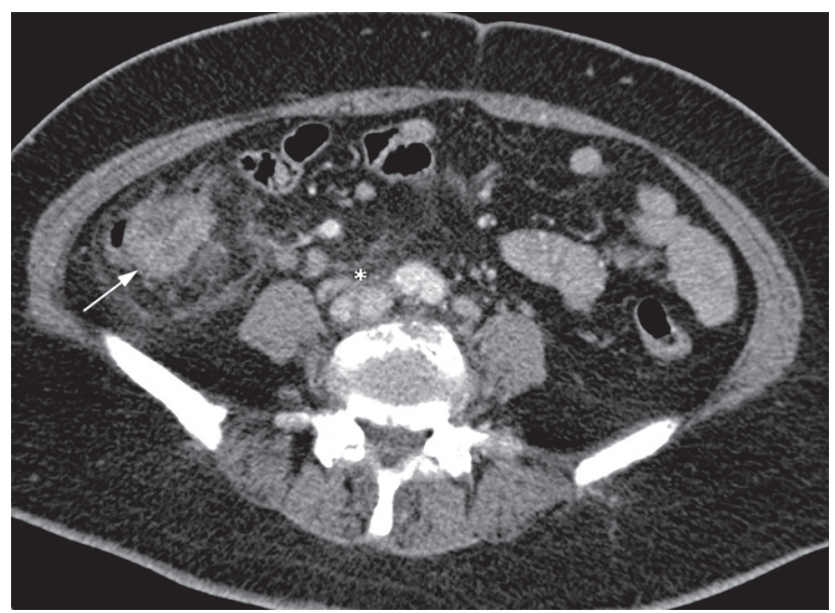

Fig. 4 Histologically proven metastasis of poorly differentiated breast adenocarcinoma to the ascending colon. Computed tomography shows short segment, irregular thickening of the ascending colon (arrow) with pericolonic fat stranding and enlarged retroperitoneal lymph nodes (asterisk), mimicking primary colonic tumor at presentation.

\section{Case 4}

A 56-year-old lady presented with abdominal pain and right breast mass. Contrast-enhanced CT scan of the abdomen and pelvis revealed short segment, irregular thickening of the ascending colon with pericolonic fat stranding and enlarged retroperitoneal lymph nodes ( - Fig. 4 ), mimicking primary colonic tumor at presentation. Investigation and biopsy of the right breast mass (not shown) showed a poorly differentiated 


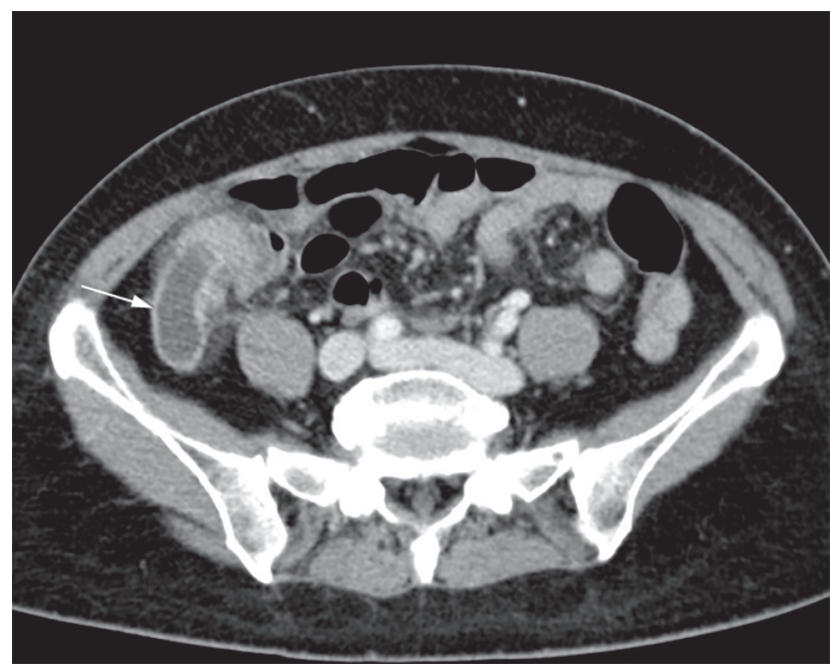

Fig. 5 Histologically proven solitary metastasis of invasive ductal breast carcinoma to the appendix. Computed tomography shows dilated and thickened appendix with periappendiceal fat stranding (arrow), mimicking acute appendicitis at presentation. ${ }^{*}$ This case has been reported separately in a case report by the author. ${ }^{5}$

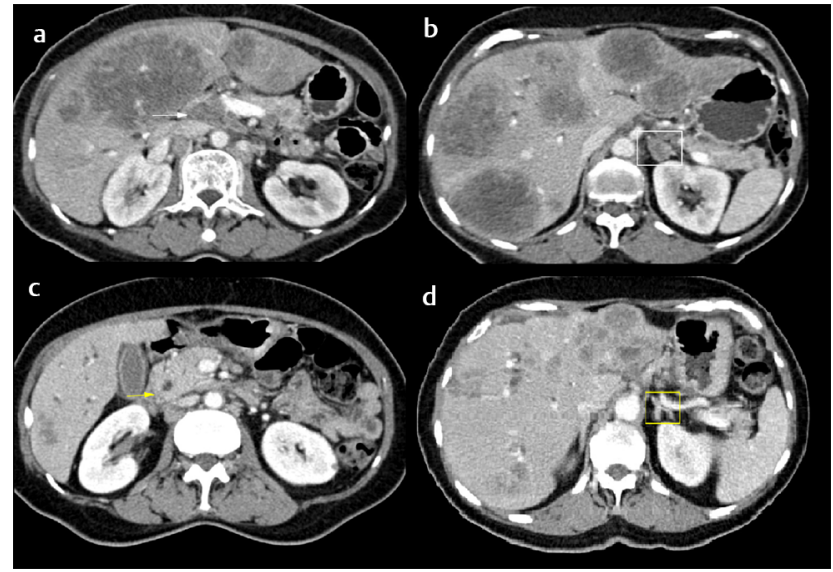

Fig. 6 Metastasis of invasive ductal breast carcinoma to pancreas. Pretreatment computed tomography (CT) (A, B) shows multiple lesions in liver, pancreatic body and tail (arrow), and adrenal gland (box). Posttreatment CT (C, D) shows improvement (yellow arrow, yellow box). Multiplicity and adrenal gland involvement favor metastases.

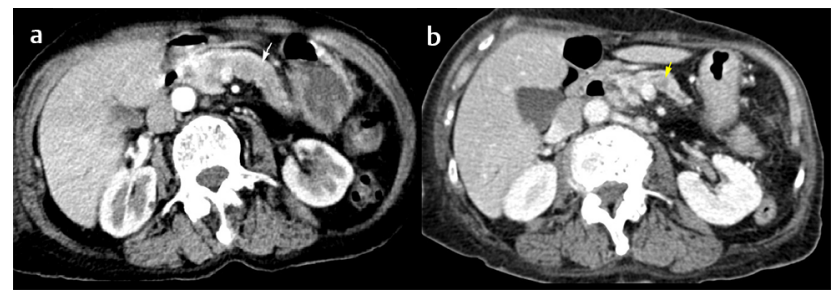

Fig. 7 Metastasis of invasive ductal breast carcinoma to the pancreas. Pretreatment computed tomography (CT) (A) shows multiple ill-defined hypodensities occupying the pancreatic neck, body, and tail (arrow). Note the absence of pancreatic-ductal dilatation and preservation of retropancreatic fat. Posttreatment CT (B) shows improvement (yellow arrow). breast adenocarcinoma and biopsy of the ascending colon revealed metastatic poorly differentiated breast adenocarcinoma of the ascending colon with a similar histological profile as the breast mass.

\section{Case 5}

A 59-year-old lady presented with the classical presentation of acute appendicitis with right iliac fossa pain and fever. She also complained of a left breast mass. Contrast-enhanced CT scan of the abdomen and pelvis revealed dilated and thickened appendix with periappendiceal fat stranding (-Fig. 5). Investigation and biopsy of the left breast mass (not shown) revealed an invasive ductal carcinoma of the breast. She underwent appendectomy and subsequent open right hemicolectomy and histology revealed metastatic invasive ductal breast carcinoma to the appendix.

\section{Pancreas-2 Cases \\ Case 6}

A 64-year-old lady presented with a prior history of stage-1 invasive ductal carcinoma of the breast. She presented with jaundice 2 years after treatment. Contrast-enhanced CT abdomen and pelvis revealed multiple hypodense lesions in the pancreatic head, body, and tail ( - Fig. $\mathbf{6 A}$ ) and coexisting metastasis to the left adrenal gland ( - Fig. 6B). Both the pancreatic and adrenal lesions improved after palliative chemotherapy ( - Fig. 6C, D).

\section{Case 7}

A 73-year-old lady presented with stage-4 invasive ductal carcinoma of the breast at diagnosis. Contrast-enhanced CT abdomen and pelvis at staging revealed multiple ill-defined hypodensities occupying the pancreatic neck, body, and tail ( - Fig. 7A) that improved after palliative chemotherapy ( - Fig. 7B).

\section{Spleen-2 Cases \\ Case 8}

A 68-year-old lady presented with stage-4 invasive ductal carcinoma of the breast at diagnosis. Contrast-enhanced CT abdomen and pelvis at staging revealed multiple ill-defined splenic hypodensities ( - Fig. 8A) that improved after pallia-

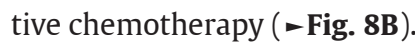

\section{Case 9}

A 54-year-old lady presented with a prior history of stage-1 invasive ductal carcinoma of the breast. She presented with shortness of breath 3 years after treatment. Contrast-enhanced CT thorax, abdomen, and pelvis showed new bilateral lung, bone, and liver metastases and also a single well-circumscribed splenic hypodensity (-Fig. 9A). The splenic lesion improved after palliative chemotherapy (-Fig. 9B). 


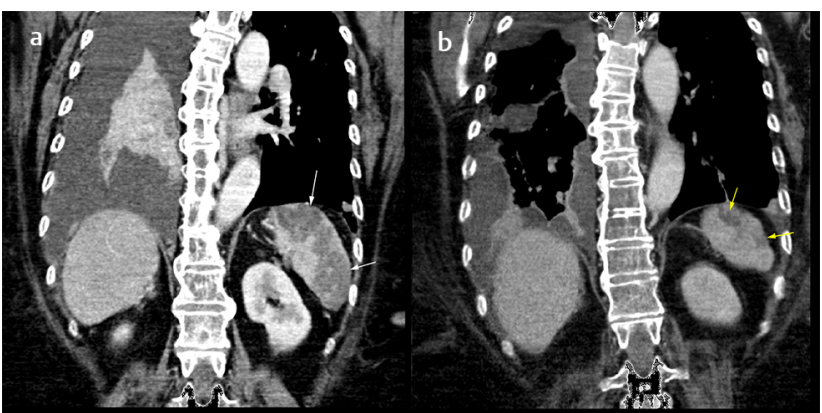

Fig. 8 Metastasis of invasive ductal breast carcinoma to the spleen. Pretreatment computed tomography (CT) (A) shows multiple large ill-defined splenic hypodensities (arrows). Right pleural effusion is also included. Posttreatment CT (B) shows improvement (yellow arrows).

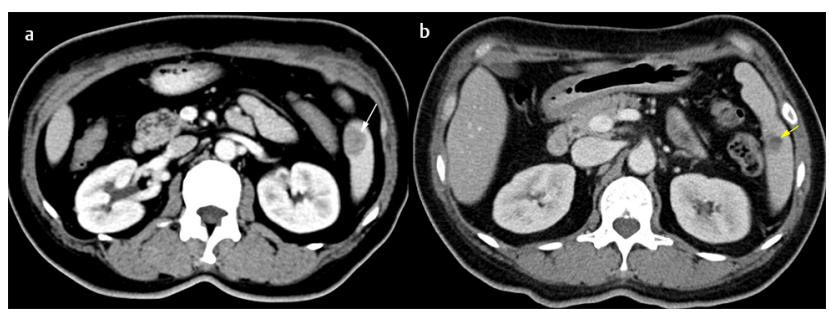

Fig. 9 Metastasis of invasive ductal breast carcinoma to the spleen. Pretreatment computed tomography (CT) image (A) shows a single well-circumscribed splenic hypodensity (arrow), a rarer presentation as compared with multiple splenic lesions. Posttreatment CT (B) shows improvement (yellow arrow).

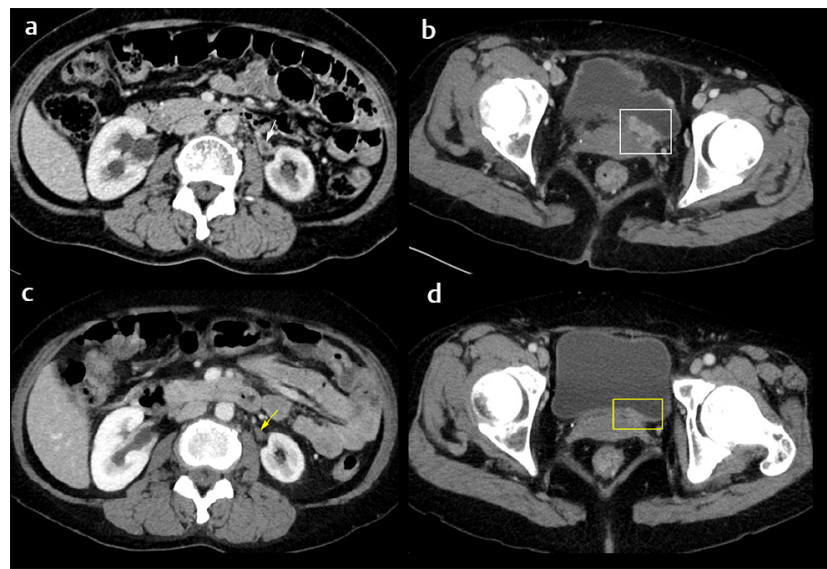

Fig. 10 Metastasis of invasive lobular breast carcinoma to the ureter. Pretreatment computed tomography (CT) (A, B) shows circumferential thickening of the left proximal ureter (arrow) and irregular mass-like thickening in the left vesicoureteric junction (box). Posttreatment CT (C, D) shows improvement (yellow arrow and yellow box).

\section{Ureter-2 Cases}

Case 10

A 56-year-old lady presented with a prior history of stage-1 invasive lobular carcinoma of the breast. She underwent mastectomy and ipsilateral axillary clearance but declined subsequent hormonal and chemotherapy. Two years later, restaging CT thorax, abdomen, and pelvis showed new metastasis to the left ureter with circumferential thickening

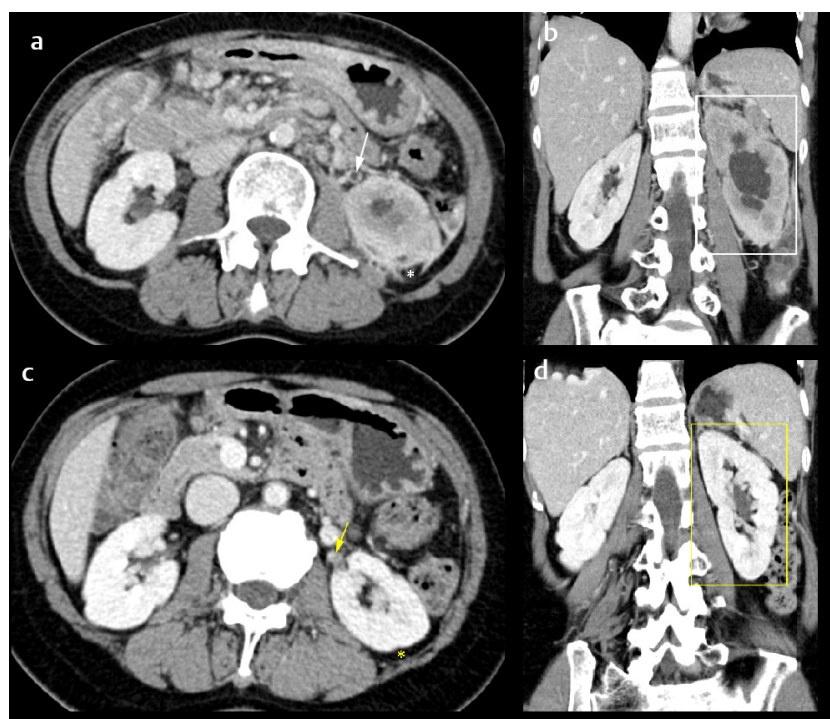

Fig. 11 Metastasis of invasive lobular breast carcinoma to ureter. Pretreatment computed tomography (CT) (A, B) shows circumferential-thickening of proximal ureter (arrow) and moderate hydronephrosis (box). Perinephric soft-tissue thickening (asterisk) is atypical of primary ureteric malignancy. Posttreatment CT (C, D) shows improvement (yellow arrow, yellow-asterisk, and yellow box).

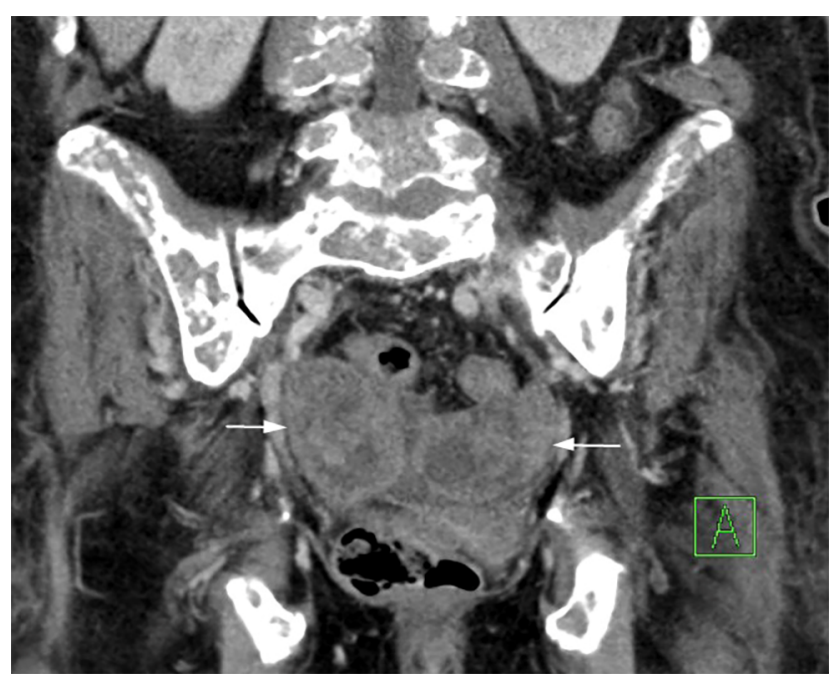

Fig. 12 Histologically proven metastasis of invasive ductal breast carcinoma to the ovaries. Computed tomography shows heterogeneously enhancing solid-cystic bilateral ovarian masses (arrows). Bony metastases are also included. Bilateral and symmetrical involvement of the ovaries favor metastases over primary ovarian malignancy.

of the left proximal ureter ( - Fig. 10A) and irregular mass-like thickening in the left vesicoureteric junction $(-$ Fig. 10B $)$ that improved after palliative chemotherapy ( - Fig. 10C, D).

\section{Case 11}

A 50-year-old lady presented with a prior history of stage-1 invasive lobular carcinoma of the breast. She presented with abdominal pain 9 years after treatment. Contrast-enhanced CT scan of the abdomen and pelvis revealed circumferential-thickening of the proximal ureter, 


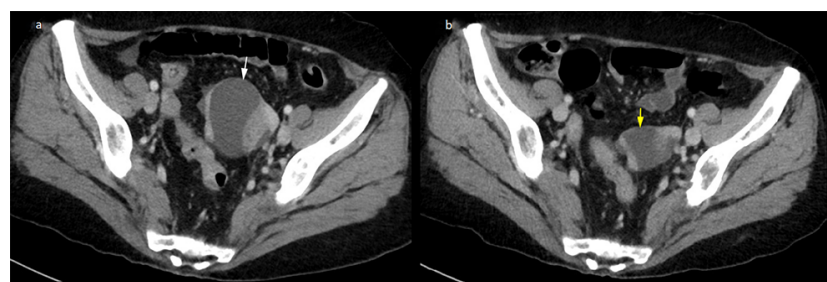

Fig. 13 Metastasis of invasive lobular breast carcinoma to the ovary. Pretreatment computed tomography (CT) (A) shows a predominantly cystic left ovarian mass with solid component (arrow). Posttreatment CT (B) shows improvement (yellow arrow).

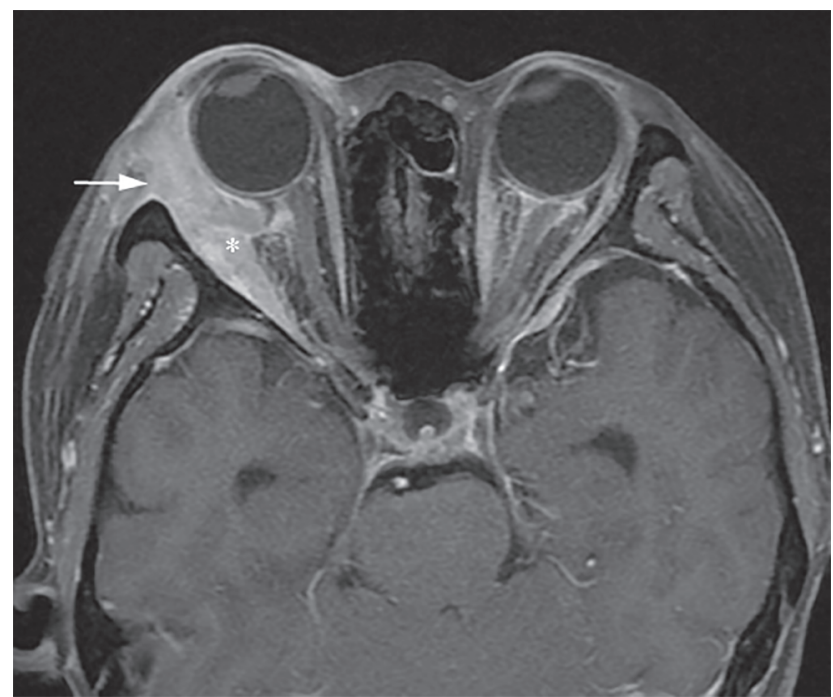

Fig. 14 Histologically proven metastasis of invasive lobular breast carcinoma to the orbit. Postcontrast T1-weighted-fat-saturated magnetic resonance imaging shows mild right proptosis, infiltrative enhancing masses in the pre- and postseptal space (arrow) and right lateral rectus muscle involvement (asterisk). Muscle involvement has been associated with metastases.

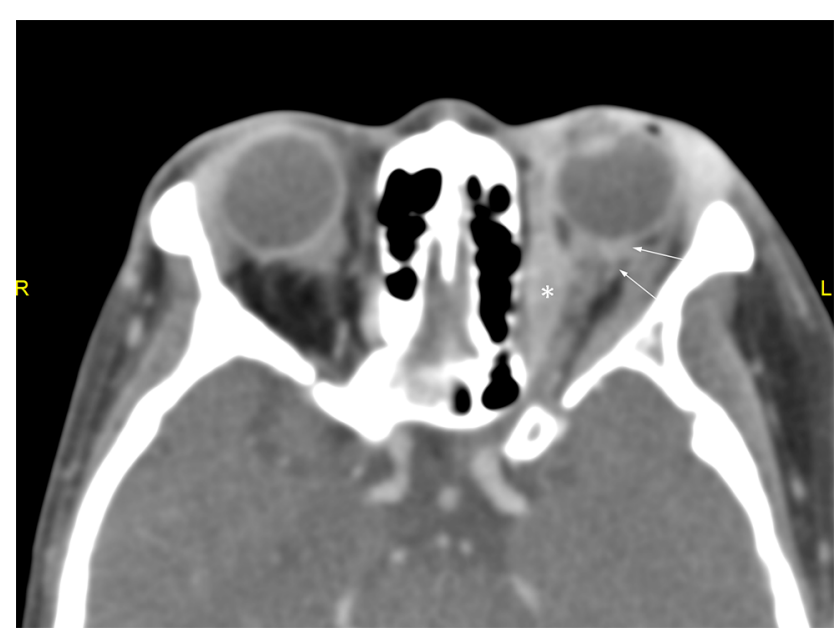

Fig. 15 Histologically proven metastasis of invasive lobular breast carcinoma to the orbit. Computed tomography shows infiltrative enhancement along the left optic-nerve-sheath complex (arrows), a feature known to be associated with breast carcinoma metastasis. Left medial rectus muscle (asterisk) and anterior orbital fat infiltration are seen. perinephric soft tissue thickening, and moderate hydronephrosis ( - Fig. 11A, B) that improved after palliative chemotherapy ( - Fig. 11C, D).

\section{Ovaries-2 Cases}

\section{Case 12}

A 52-year-old lady presented with a history of stage- 1 invasive ductal carcinoma of the breast. She presented with abdominal pain 10 years after treatment. Contrast-enhanced CT abdomen and pelvis showed heterogeneously enhancing solid-cystic bilateral ovarian masses (-Fig. 12). CA-125 was normal, whereas CA 15-3 was markedly elevated at presentation. Biopsy of the right ovarian mass revealed metastatic invasive ductal carcinoma of the breast to the ovary.

\section{Case 13}

A 56-year-old lady presented with a history of stage-1 invasive lobular carcinoma of the breast who declined treatment. Two years later, restaging CT thorax, abdomen, and pelvis showed a new predominant cystic left ovarian mass ( - Fig. 13A) that improved after palliative chemotherapy (-Fig. 13B).

\section{Orbits-2 Cases \\ Case 14}

A 50-year-old lady presented with a history of stage-1 invasive lobular carcinoma of the breast. She presented with right orbital swelling 9 years after treatment. Contrast-enhanced MRI orbits showed mild right proptosis and infiltrative enhancing masses in the pre- and postseptal space involving the right lateral rectus muscle ( $\mathbf{- F i g . 1 4}$ ). Biopsy of the right orbit revealed metastatic invasive lobular breast carcinoma to the orbit.

\section{Case 15}

A 56-year-old lady presented with a history of stage-1 invasive lobular carcinoma of the breast. She presented with left eye swelling 2 years after the surgery. Contrast-enhanced CT orbits showed infiltrative soft tissue involvement along the left optic-nerve-sheath complex, left medial rectus muscle, and anterior orbital fat ( - Fig. 15). Biopsy of the anterior orbital soft tissue yielded metastatic invasive lobular carcinoma of the breast to the orbit.

\section{Discussion}

Less than $1 \%$ of breast carcinomas metastasize to the GIT. Invasive lobular carcinomas have been reported to have greater propensity to metastasize to the GIT compared with invasive ductal carcinomas. The common sites of involvement include stomach and small and large bowel. ${ }^{3-5}$

The commonest appearance of metastatic carcinoma, especially of the lobular subtype, is tumor infiltration in submucosa or muscularis propria resulting in linitis plastica for stomach metastases and long-segment concentrate mural thickening for bowel metastases as demonstrated in our case 2 (-Fig. 2). ${ }^{3}$ When present, sparing of the mucosa, as illustrated in our case 3 ( $\mathbf{- F i g . 3}$ ), is deemed highly suggestive of 
breast metastasis to the rectum and can help to differentiate metastasis from primary rectal tumor. ${ }^{4}$ Other differentiating imaging features include marked T2 hypointensity ( - Fig. $\mathbf{3}$ ), mild restricted diffusion, and relative absence of mesorectal or pelvic sidewall nodal disease, despite extensive involvement of the bowel wall. ${ }^{4}$ Though rare, focal wall thickening has been described and was seen in our case 1 ( - Fig. 1). We acknowledge that it is, however, sometimes impossible to differentiate metastatic etiology from the primary tumor (-Fig. 4) or infection/inflammation based on imaging alone ( - Fig. 5). ${ }^{5}$ This observation is evidenced by our cases 4 and 5 .

The pancreas is a rare site for metastases, accounting for only $2 \%$ of pancreatic malignancies. ${ }^{6}$ The commonest primary malignancy to metastasize to pancreas is renal cell carcinoma (62\%), followed by nonsmall-cell lung cancer, colon, and stomach. Pancreatic metastases from breast cancer are very rare but, if present, usually reflect high tumor burden and are indicative of poor prognosis. ${ }^{\text {? }}$

Some authors opine that breast metastases may appear more vascular than primary pancreatic adenocarcinoma. ${ }^{7}$ In an attempt to differentiate metastases to the pancreas from primary pancreatic tumor, several features have also been suggested. First, multiplicity of lesions within the pancreas ( $\mathbf{- F i g . ~ 6 )}$ is relatively common in pancreatic metastases but is atypical of a primary pancreatic tumor. Second, coexisting metastases at sites that are not typically involved by primary pancreatic adenocarcinoma, such as the bone or adrenal glands ( - Fig. 6), should also suggest the possibility of metastatic disease of the pancreas. These two features were demonstrated in case 6 . Lastly, similar to case 7 , the absence of biliary duct dilatation and preservation of the retropancreatic fat in cases of large pancreatic tumor favor metastasis (-Fig. 7). ${ }^{6}$

Spleen is a rare metastatic site for solid tumor and, if present, is usually accompanied by disseminated visceral metastases. Studies revealed that metastasis to spleen occurs only in $3 \%$ of solid tumors, of which lung, cutaneous malignant melanoma, and breast cancer are the most frequent primaries. ${ }^{8}$

Some authors hypothesize that abundance of immunological monitoring and production of angiostatin, an antiangiogenic factor, may explain the relative resistance of spleen to implantation of tumor cells. Other anatomical factors such as the sharp angle of the splenic artery (making it difficult for tumor emboli to enter the spleen) and the rhythmic contractile nature of the spleen have also been proposed. ${ }^{8,9}$

Metastatic foci in spleen often appear as ill-defined low-density masses on CT, as seen in case 8 ( $\bullet$ Fig. 8). ${ }^{9}$ Though commonly multiple, a single well-circumscribed hypodense lesion has also been described and was encountered in case 9 ( - Fig. 9). ${ }^{8}$ Ultrasound can show both hypoechoic and hyperechoic patterns. ${ }^{9}$

About $90 \%$ of ureteric malignancies are transitional cell carcinoma of the ureter. Metastases to the ureters are rare. About 7.8\% of ureteric metastases were from primary breast malignancy with only a handful of case reports recorded in the literature. The presence of ureteral metastases signifies advanced disease. ${ }^{10,11}$
The differentiation of ureteral metastasis from primary urothelial tumors is difficult based on imaging alone. Asymmetric wall enhancement, urothelial wall thickening ( - Figs. 10 and 11 ), and urinary obstruction ( - Fig. 11) are expected imaging findings. Some authors suggest that primary urothelial tumors tend to involve a long segment, whereas secondary tumors usually involve a relatively short segment. Perinephric infiltration, which is demonstrated in case 11 ( - Fig. 11), is atypical of primary ureteric malignancy. Lymphoma affecting the urinary system does not typically cause obstruction ( $\mathbf{- F i g . ~ 1 1 ) . ~ M u l t i f o c a l i t y ~ d o e s ~}$ not seem to be a differentiating factor. Differential diagnoses include strictures secondary to stone passage and other benign etiology such as tuberculosis, amyloidosis, and endometriosis. ${ }^{11}$

A range of prevalence from 3 to $38 \%$ has been reported for metastases and micrometastases to the ovaries. The most common primary source is the gastrointestinal system followed by breast cancer, with invasive lobular carcinomas having as much as three times greater tendency to metastasize to the ovaries compared with the other subtypes of breast cancers. ${ }^{3,12,13}$

However, one should bear in mind that patients with a history of breast cancer are three to seven times more likely to develop primary ovarian cancer as opposed to ovarian metastases. ${ }^{12}$ To differentiate between these two, several factors have been proposed. Large size of the primary breast tumor, small bilateral ovarian masses ( - Fig. 12), concurrent widespread metastases, and minor elevation of serum CA-125 level $(<80 \mathrm{U} / \mathrm{mL})$ but marked elevation of CA 15-3 $(>100 \mathrm{U} / \mathrm{mL})$, all of which are featured in case 12 , are deemed to favor metastases to the ovary rather than primary ovarian malignancy. ${ }^{12,13}$

Conversely, unilateral ovarian mass, absence of other site of involvement, markedly elevated serum CA-125 level, and positive family history of primary ovarian cancer are suggestive of primary ovarian malignancy. ${ }^{12,13}$

The appearance of metastases to ovary can range from solid and solid-cystic ( - Fig. 12) to predominantly cystic (-Fig. 13). . $^{1213}$

The incidence of metastases to the orbit among patients with breast carcinoma varies between 8 and $10 \%$. Of all metastatic tumors to the orbit, breast carcinoma is the commonest primary tumor, contributing between 29 and $51 \%$ of all orbital metastases. ${ }^{14}$

Metastases may involve any part of the orbit but are more common in the extraconal space and the anterior orbit. $^{14,15}$ Imaging findings range from a focal mass to a diffusely infiltrating lesion (-Figs. 14 and $\mathbf{1 5}$ ). When present, enophthalmos is thought to be typical of scirrhous breast carcinoma metastasis, postulated to be related to ocular retraction resulting from the fibrotic nature of the condition. Other features such as a muscle involvement (-Figs. 14 and 15) and osseous destruction have also been described to be associated with metastases. ${ }^{14}$ Tumor infiltration of the optic nerve and sheath, as demonstrated in our case 15 (-Fig. 15), is also known to be a feature of breast carcinoma metastasis. $^{15}$ 
Lymphoma is the main differential on imaging. However, patients with lymphoma would typically present with proptosis rather than enophthalmos. ${ }^{14}$

\section{Conclusion}

Unusual locations of metastatic breast cancer can present as a diagnostic conundrum, with the main differential being that of a synchronous primary tumor in the affected organ. Breast metastases may occur years after the initial diagnosis and treatment of the primary tumor. Several differentiating features have been proposed and our case series summarize the available features. We recognize that although some of the radiological findings are not specific to breast metastases, awareness of the varied manifestation of disease, diligent comparison with prior imaging studies, and checking for a history of breast malignancy are invaluable in guiding the radiologist to the correct diagnosis. The gold standard for diagnosis ultimately lies upon histological correlation.

Metastases in unusual locations often happen in the advanced stage of breast cancer with multiorgan metastases. We are aware that in clinical practice, once a metastatic disease is documented, biopsy of additional lesions may not be and is often not performed. Additional factors such as patient's physical fitness, patient's choice, accessibility of the diseased organ, and response to treatment often influence the clinical decision of whether a tissue diagnosis is ultimately required. A collaboration of clinical, laboratory, imaging, and histopathological findings and trial of treatment are often needed for diagnosis. Treatment plans should also be made through close cooperation with the patient and a multidisciplinary team involving the surgeon, oncologist, radiation oncologist, and radiologist. $4,6,11,12$

\section{Financial Support and Sponsorship}

Nil.

\section{Conflicts of Interest}

There are no conflicts of interest.
Australia, 2001-2016: a population-based health record linkage study protocol. BMJ Open 2019;9(2):e02641410.1136/ bmjopen-2018-026414

2 Odele P, Khan SA, 67 - Management of the intact breast primary in the setting of metastatic disease. In: Bland KI, Copeland EM, Klimberg VS, Gradishar WJ, eds. The Breast. $5^{\text {th }}$ ed. Philadelphia, PA: Elsevier; 2018 867-875.e2

3 Winston CB, Hadar O, Teitcher JB, Caravelli JF, Sklarin NT, Panicek DM, et al. Metastatic lobular carcinoma of the breast: patterns of spread in the chest, abdomen, and pelvis on CT. AJR Am J Roentgenol 2000;175(3):795-800

4 Lau LC, Wee B, Wang S, Thian YL. Metastatic breast cancer to the rectum: a case report with emphasis on MRI features. Medicine (Baltimore 2017;96(17):e673910.1097/ MD.0000000000006739

5 Chotai N. Acute appendicitis in a patient with breast mass: is there anything fishy? Breast J 2018;24(6):1074-1075

6 Triantopoulou C, Kolliakou E, Karoumpalis I, Yarmenitis S, Dervenis C. Metastatic disease to the pancreas: an imaging challenge. Insights Imaging 2012;3(2):165-172

7 Tunio MA, Fatani H, Riaz K, AlAsiri M. Pancreatic metastasis of breast cancer: a rare cause of obstructive jaundice. J Dow Univ Health Sci 2015;9:39-40

8 Iype S, Akbar MA, Krishna G. Isolated splenic metastasis from carcinoma of the breast. Postgrad Med JNippon Igaku Hoshasen Gakkai Zasshi 2002;78(917):173-174Imada H, Nakata H, Horie A. 1991;51(5):498-503

9 Gabsi A, Yahiaoui Y, Zenhani A, Herbegue K, Meddeb K, Mokrani A,et al. Ureteral metastasis in carcinoma of the breast. Urol Case Rep 2018;21:38-40

10 Karaosmanoglu AD, Onur MR, Karcaaltincaba M, Akata D, Ozmen MN. Secondary tumors of the urinary system: an imaging conundrum. Korean J Radiol 2018;19(4):742-751

11 Tian W, Zhou Y, Wu M, Yao Y, Deng Y. Ovarian metastasis from breast cancer: a comprehensive review. Clin Transl Oncol 2019;21(7):819-827

12 Bigorie V, Morice P, Duvillard P, Antoine M, Cortez A, Flejou JF, et al. Ovarian metastases from breast cancer: report of 29 cases. Cancer 2010;116(4):799-804

13 Meltzer DE, Chang AHY, Shatzkes DR. Case 152: orbital metastatic disease from breast carcinoma. Radiology 2009;253(3):893-896

14 Müller-Forell W, Pitz S, Orbital pathology. In: Baert AL, Sartor K, Müller-Forell WS, eds. Imaging of Orbital and Visual Pathway Pathology. Medical Radiology. Berlin, Heidelberg: Springer; 2002 147-340

\section{References}

1 Lord SJ, Kiely BE, Pearson SA, Daniels B, O'Connell D, Beith J, et al. Metastatic breast cancer incidence, site and survival in 